UVX 2008 (2009) 183-187

(C) EDP Sciences, 2009

DOI: $10.1051 / \mathrm{uvx} / 2009030$

\title{
Stabilité thermique des performances spectrales de miroirs EUV
}

\author{
C. Hecquet ${ }^{1}$, F. Delmotte ${ }^{1}$, M. Rouillay ${ }^{2}$, A. Rinchet ${ }^{3}$, F. Varnière ${ }^{1}$, \\ E. Melchakov ${ }^{1}$ et M.-F. Ravet-Krill ${ }^{1}$ \\ ${ }^{1}$ Laboratoire Charles Fabry de l'Institut d'Optique, Campus Polytechnique, 91127 Palaiseau, \\ France \\ ${ }^{2}$ Laboratoire d'Interaction du Rayonnement X avec la Matière, Bât. 350, 91405 Orsay, \\ France \\ ${ }^{3}$ Sagem DS, Avenue de la Tour Maury, 91280 St. Pierre du Perray, France
}

\begin{abstract}
Résumé. Le développement de miroirs EUV résistant à des températures élevées représente un enjeu important pour la lithographie EUV. Nous avons optimisé et déposé plusieurs empilements multicouches $\mathrm{Mo} / \mathrm{SiC} / \mathrm{Si} / \mathrm{SiC}$ en faisant varier les épaisseurs respectives de $\mathrm{SiC}$ aux deux interfaces. Ces différents types d'empilements ont ensuite subi une série de recuits thermiques pour évaluer l'influence des deux paramètres temps et température de recuit, sur les performances spectrales. Des structures stables après un recuit de $200 \mathrm{~h}$ à $400^{\circ} \mathrm{C}$ ont été optimisées et caractérisées.
\end{abstract}

\section{INTRODUCTION}

Les nouvelles générations de source plasma laser EUV pour la photolithographie à 13,5 nm permettent d'obtenir des intensités de plus de $1000 \mathrm{~W} / 2 \pi$ sr. Les traitements réfléchissants des optiques de collection du flux lumineux sont actuellement un facteur limitant pour les performances et la durée de vie de ces sources. Les principales problématiques à résoudre sont l'érosion par les particules rapides, la contamination surfacique des miroirs multicouches [Si/Mo] par les débris des matériaux de la source ( $\mathrm{Sn}$ ou $\mathrm{Li}$ ) et la contamination interne induite par la diffusion dans l'empilement des polluants précités. À chaque technologie de source est associée une technique de limitation de contamination mais aussi une technique de nettoyage des revêtements. Une des solutions consiste à limiter les dépôts de matériaux polluants sur la surface des miroirs en chauffant ces miroirs à des températures supérieures à $400^{\circ} \mathrm{C}$. De précédentes études ont montré que l'ajout de «barrières antidiffusion » en matériaux carbonés $\left(\mathrm{B}_{4} \mathrm{C}, \mathrm{C}\right.$, $\mathrm{SiC}$ ) entre chacune des couches minces Molybdène et Silicium procure une meilleure stabilité thermique de l'empilement multicouches [1]. De plus, ces matériaux présentent une vitesse d'érosion plus faible que le Mo ou Si ce qui permet d'augmenter la durée de vie des revêtements en conditions extrêmes. L'objectif de notre étude est de déterminer les épaisseurs optimales de couches barrières aux deux interfaces afin d'obtenir des miroirs EUV résistants à des températures élevées en utilisation prolongée.

\section{SIMULATION ET DÉPÔT DES MIROIRS Mo/Si AVEC COUCHES BARRIÈRES EN SiC}

\subsection{Optimisation de la réflectivité des multicouches}

D'un coté, les multicouches [a-Si/Mo] présente une réflectivité optimale $(0,74$ en théorie) mais leur stabilité thermique est limitée (forte dégradation au delà de $150^{\circ} \mathrm{C}$ ). D'un autre coté, les multicouches [a-SiC/Mo] ont montré de très bonnes qualités de tenue à $400^{\circ} \mathrm{C}$ et $500^{\circ} \mathrm{C}$ avec un temps de stabilisation déterminé mais une réflectivité inférieure à 0,54 . L'ajout de a-SiC aux interfaces de la structure [a$\mathrm{Si} / \mathrm{Mo}]$ représente un compromis intéressant entre ces deux systèmes. Dans cette étude, nous nous intéressons donc aux multicouches de type [a-SiC/a-Si/a-SiC/Mo] dans le but d'optimiser la réflectivité tout en assurant une bonne tenue en température. Les couches de a-SiC aux interfaces Mo sur a-Si et 


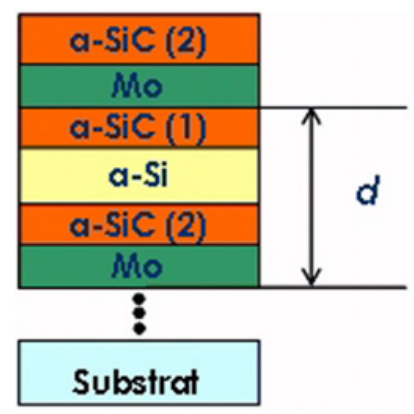

Figure 1. Schéma des miroirs multicouches $\mathrm{Mo} / \mathrm{a}-\mathrm{Si}$ avec couches barrières en a-SiC.

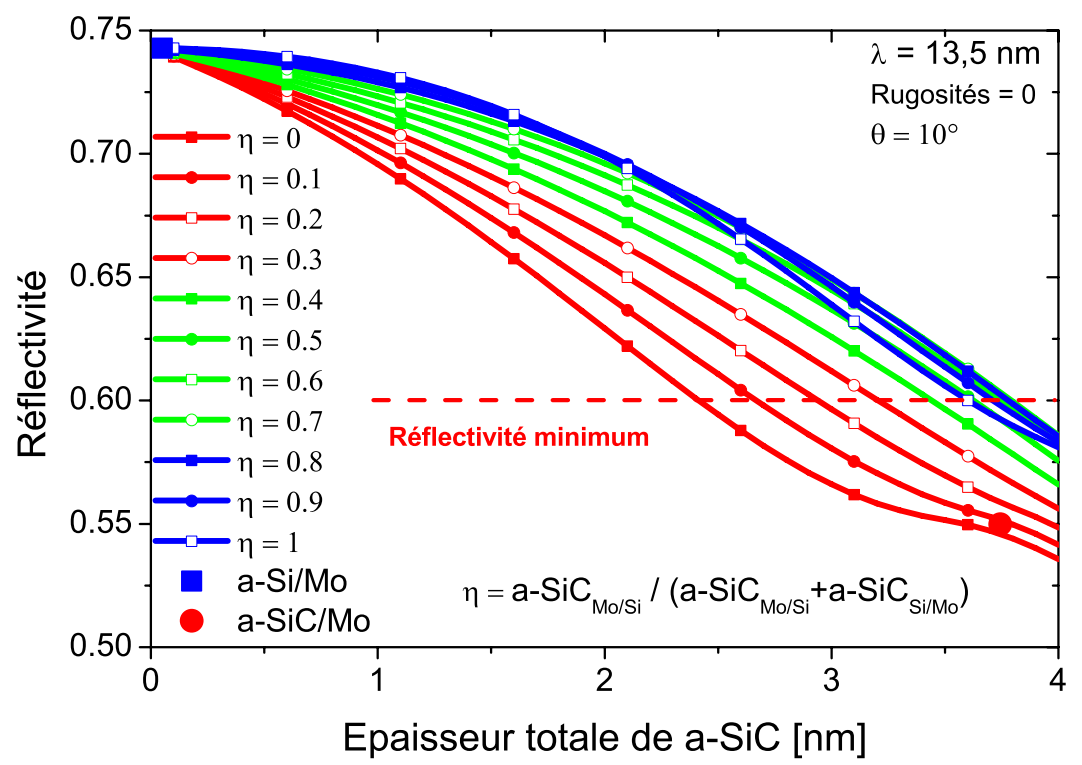

Figure 2. Réflectivité simulée à $\lambda=13,5 \mathrm{~nm}$ pour des multicouches [a-SiC/a-Si/a-SiC/Mo] en fonction de l'épaisseur totale de a-SiC et du paramètre $\eta$.

a-Si sur Mo seront notées respectivement a-SiC(1) et a-SiC(2) (voir figure 1). Dans un premier temps, nous avons étudié l'influence de l'épaisseur totale de a-SiC et de sa répartition entre les deux interfaces sur la réflectivité théorique à $\lambda=13,5 \mathrm{~nm}$. Les résultats des simulations sont représentés sur la figure 2. Nous avons défini un paramètre $\eta$ qui représente la répartition de a-SiC entre les deux interfaces :

$$
\eta=\text { paisseur[a-SiC(1)]/(paisseur[a-SiC(1)] + paisseur[a-SiC(2)]) }
$$

Ces simulations montrent qu'il est préférable, pour conserver un bon pouvoir réflecteur, de choisir ce paramètre $\eta$ supérieur ou égal à 0,5 .

\subsection{Dispositifs expérimentaux}

Les simulations précédentes nous ont permis de définir plusieurs structures multicouches pour l'étude de leur stabilité thermique. Nous avons choisi six structures avec des épaisseurs totales de a-SiC comprises entre $0,9 \mathrm{~nm}$ et $2,4 \mathrm{~nm}$ et des paramètres compris entre 0,5 et 0,63 . Toutes les multicouches possèdent 50 périodes et les épaisseurs de Mo et $\mathrm{Si}$ ont été calculées afin d'optimiser la réflectivité de l'empilement à $\lambda=13,5 \mathrm{~nm}$. Les multicouches ont été déposées à l'aide d'un réacteur de dépôt 


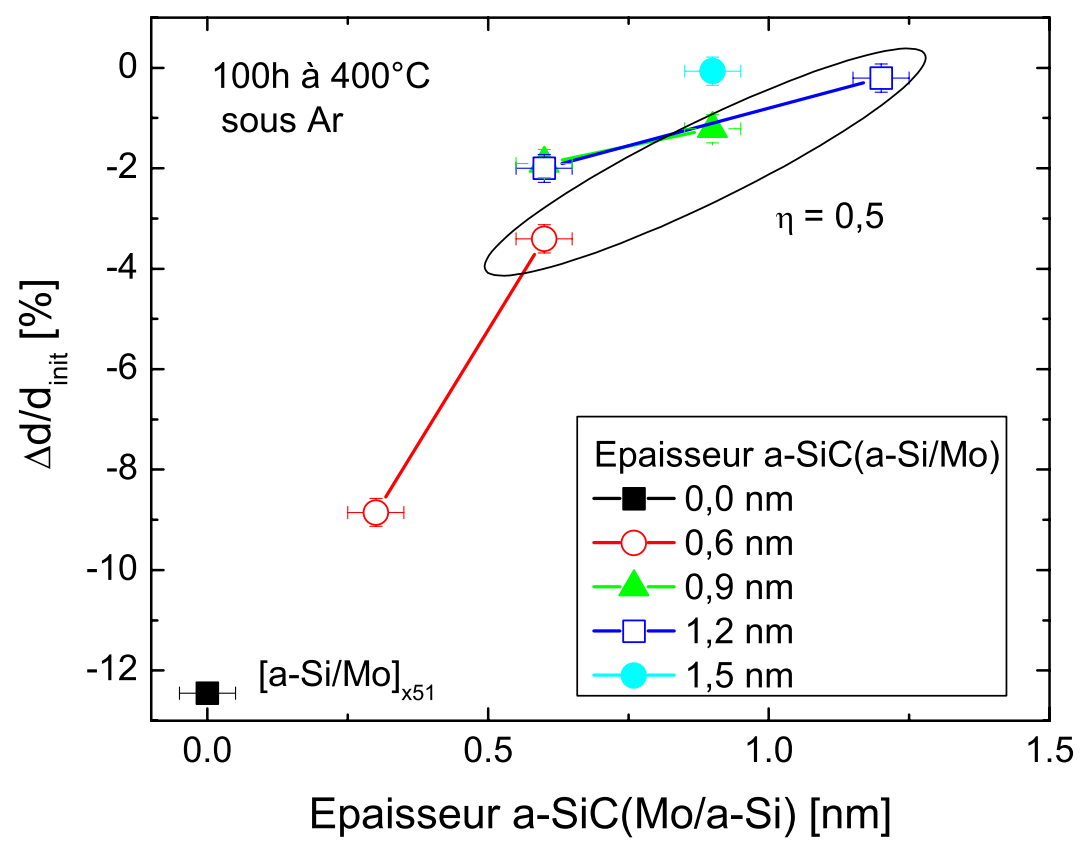

Figure 3. Influence des épaisseurs d'a-SiC aux interfaces sur la stabilité de la période d de l'empilement $100 \mathrm{~h}$ à $400{ }^{\circ} \mathrm{C}$ sous Ar.

par pulvérisation cathodique magnétron décrit dans [2] et [3]. Après chaque dépôt, les multicouches sont analysées par réflectométrie en $\mathrm{X}$ rasant à $\lambda=0,154 \mathrm{~nm}$. L'ajustement de la courbe expérimentale permet de déterminer la période de l'empilement, l'épaisseur des différentes couches, l'indice complexe des matériaux à cette longueur d'onde ainsi que la rugosité aux interfaces [4]. Pour caractériser les multicouches à la longueur d'onde d'utilisation, nous avons mesuré les réflectivités des multicouches à l'aide du réflectomètre de la ligne BEAR du rayonnement synchrotron d'Elettra sous un angle d'incidence de $10^{\circ}$ en polarisation $\mathrm{S}$ [5]. Nous avons également utilisé le réflectomètre à source plasma laser de la centrale CEMOX [6].

\section{3. ÉTUDE DE LA STABILITÉ THERMIQUE DES MIROIRS Mo/Si AVEC COUCHES BARRIÈRES EN SiC}

Nous avons tout d'abord étudié l'effet d'un recuit de $100 \mathrm{~h}$ à $400^{\circ} \mathrm{C}$ sous atmosphère d'argon sur l'ensemble des échantillons. La figure 3 représente la variation de période $\mathrm{d}$ normalisée à la période initiale $\mathrm{d}_{\text {init }}$ en fonction de l'épaisseur de a-SiC aux deux interfaces. L'axe des abscisses représente l'épaisseur de la barrière a-SiC à l'interface Mo/a-Si des différents échantillons. Les échantillons sont regroupés en fonction de l'épaisseur de la barrière a-SiC à l'interface a-Si/Mo. De manière générale, on constate que la structure a-Si/Mo sans barrière est la moins stable et que la stabilité de la multicouche augmente avec l'épaisseur totale de $\mathrm{SiC}$ aux interfaces. Pour les 2 structures ayant un total de 2,4 nm d'épaisseur d'a-SiC par période, la contraction de période après $100 \mathrm{~h}$ à $400{ }^{\circ} \mathrm{C}$ est inférieure à $1 \%$. La meilleure stabilité est obtenue pour la multicouche possédant $1,5 \mathrm{~nm}$ de a-SiC à l'interface a-SiC(1) et $0,9 \mathrm{~nm}$ à l'interface a-SiC(2). Les spectres de réflectivité EUV mesurés sur cette structure optimale sont tracés sur la figure 4 , avant recuit, après recuit de $100 \mathrm{~h}$ à $400^{\circ} \mathrm{C}$ et à $500^{\circ} \mathrm{C}$. Ces mesures confirment que le décalage spectral est bien inférieur à la largeur du pic de Bragg à $400^{\circ} \mathrm{C}$ mais également à $500^{\circ} \mathrm{C}$. Par ailleurs, nous pouvons constater une légère baisse de refléctivité après recuit à $400^{\circ} \mathrm{C}$. 


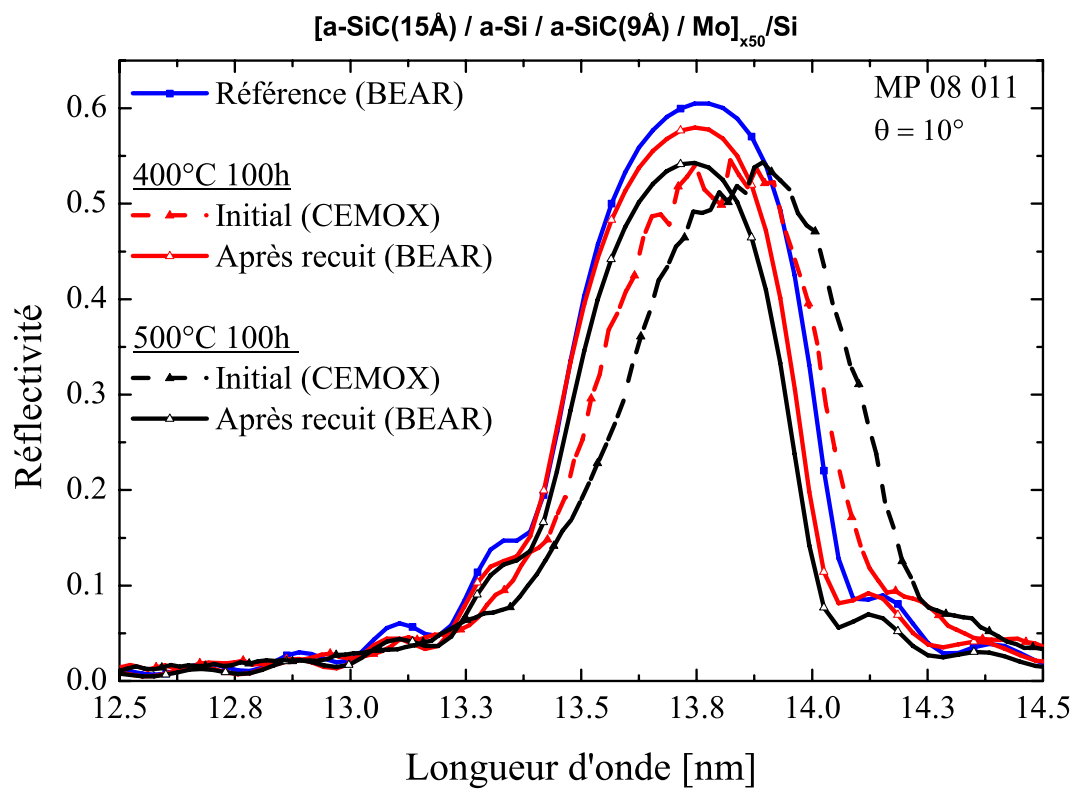

Figure 4. Spectre de réflectivité EUV d'une multicouche [a-SiC/a-Si/a-SiC/Mo] avant recuit mesurée sur CEMOX (pointillé) puis après recuit un recuit de $100 \mathrm{~h}$ sous Ar mesurée sur rayonnement synchrotron à $400^{\circ} \mathrm{C}$ (rouge) et $500^{\circ} \mathrm{C}$ (noir).

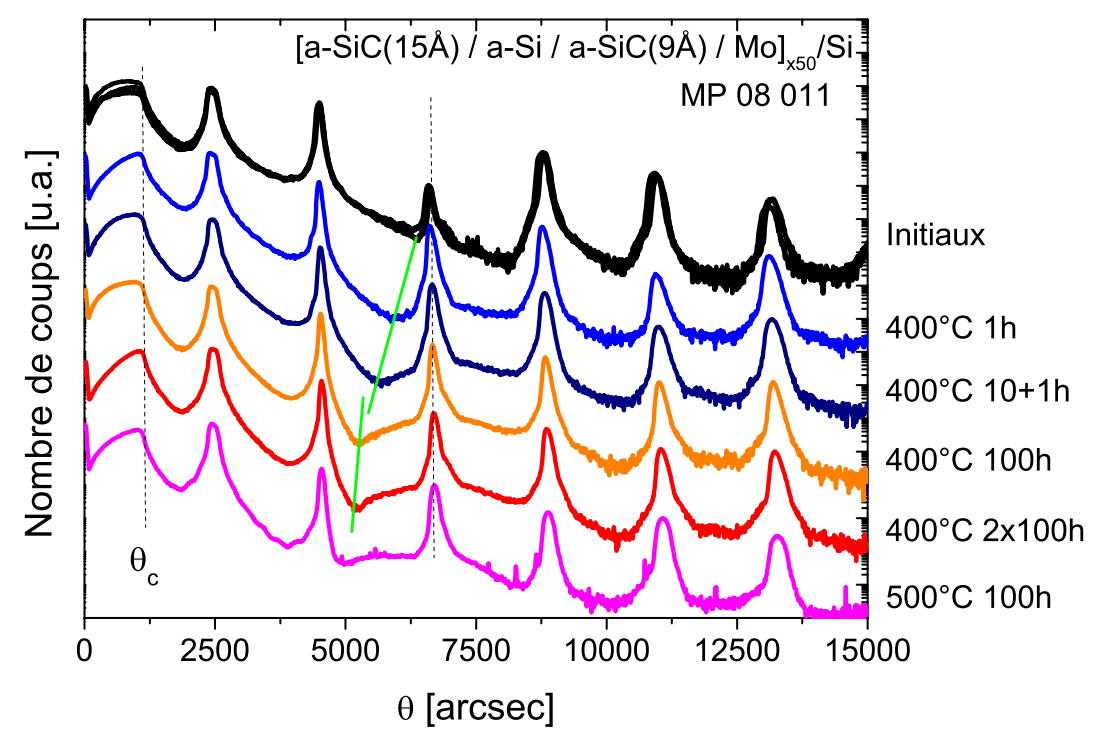

Figure 5. Influence du temps et de la température de recuit sur les mesures de réflectivité en X-rasant.

Cette baisse de réflectivité s'accentue après un recuit à $500^{\circ} \mathrm{C}$. Ces résultats peuvent s'expliquer par une modification de l'empilement multicouche sous l'effet de la température. Les mesures en réflectométrie X-rasants (figure 5) montrent en effet que l'intensité relative des pics de Bragg fluctue en fonction du temps de recuit. Ceci indique une modification des épaisseurs et/ou indices relatifs des différentes couches composant l'empilement. Ces résultats confirment aussi le fait que la structure reste périodique 
(la largueur des pics de Bragg est conservée) et que la période est stable (la position des pics de Bragg reste identique) après $200 \mathrm{~h}$ de recuit à $400^{\circ} \mathrm{C}$.

\section{CONCLUSION}

Nous avons étudié l'influence de l'épaisseur et de la répartition des couches barrières de a-SiC aux interfaces sur la stabilité thermique et la réflectivité des miroirs multicouches [a-SiC/a-Si/a-SiC/Mo]. Nous avons montré que la stabilité thermique de ces multicouches s'améliore lorsque l'on augmente l'épaisseur totale de a-SiC et qu'une reflectivité élevée peut être obtenue en minimisant l'épaisseur de a-SiC à l'interface Mo sur Si. La combinaison des mesures de réflectivité EUV et des mesures de réflectométrie en X-rasants nous a permis d'optimiser une structure stable après un recuit à $400{ }^{\circ} \mathrm{C}$ pendant $200 \mathrm{~h}$.

\section{Remerciements}

Les auteurs tiennent à remercier l'équipe de la ligne BEAR du synchrotron Elettra pour les mesures de réflectivité EUV.

\section{Références}

[1] C. Hecquet, M.F. Ravet-Krill, F. Delmotte, A. Jerome, A. Hardouin, F. Bridou, F. Varniere, M. Roulliay, F. Bourcier, J.M. Desmarres et al., Damage to VUV, EUV, and X-ray Optics 6586(1), 65860X ( 10) (2007), http://link.aip.org/link/?PSI/6586/65860X/1

[2] F. Delmotte, J. Gautier, M. Ravet, F. Bridou, A. Jerome, Journal de Physique IV (Proceedings) 127, 69 (2005), http://dx.doi.org/10.1051/jp4:2005127011

[3] J. Gautier, F. Delmotte, M. Roulliay, F. Bridou, M.F. Ravet, A. Jérome, Applied Optics 44, 384 (2005)

[4] F. Bridou, B.A. Pardo, Journal Of Optics-Nouvelle Revue D Optique 21(4), 183 (1990)

[5] S. Nannarone, F. Borgatti, A. Deluisa, B.P. Doyle, G.C. Gazzadi, A. Giglia, P. Finetti, N. Mahne, L. Pasquali, M. Pedio et al., 705, 450 (2004)

[6] C. Hecquet, M. Roulliay, F. Delmotte, M.F. Ravet-Krill, A. Hardouin, M. Idir, Journal de Physique IV 138, 259 (2006) 